\title{
Prey Foraging Behavior After Predator Introduction Is Driven by Resource Knowledge and Exploratory Tendency
}

\author{
Chloe Bracis ${ }^{1,2 *}$ and Aaron J. Wirsing ${ }^{3}$ \\ ${ }^{1}$ Université Grenoble Alpes, TIMC-IMAG/MAGE, Grenoble, France, ${ }^{2}$ Quantitative Ecology and Resource Management, \\ University of Washington, Seattle, WA, United States, ${ }^{3}$ School of Environmental and Forest Sciences, University of \\ Washington, Seattle, WA, United States
}

\section{OPEN ACCESS}

Edited by:

Tal Avgar

Utah State University, United States

Reviewed by:

Bram Van Moorter

Norwegian Institute for Nature

Research (NINA), Norway

Dan MacNulty,

Utah State University, United States

*Correspondence:

Chloe Bracis

chloe.bracis@mail.com orcid.org/0000-0003-4058-7638

Specialty section:

This article was submitted to Behavioral and Evolutionary Ecology,

a section of the journal

Frontiers in Ecology and Evolution

Received: 21 April 2021

Accepted: 06 September 2021

Published: 06 October 2021

Citation:

Bracis C and Wirsing AJ (2021) Prey Foraging Behavior After Predator Introduction Is Driven by Resource Knowledge and Exploratory Tendency.

Front. Ecol. Evol. 9:698370. doi: 10.3389/fevo.2021.698370
Predator reintroductions are often used as a means of restoring the ecosystem services that these species can provide. The ecosystem consequences of predator reintroduction depend on how prey species respond. Yet, to date, we lack a general framework for predicting these responses. To address this knowledge gap, we modeled the impacts of predator reintroduction on foragers as a function of predator characteristics (habitat domain; i.e., area threatened) and prey characteristics (knowledge of alternative habitat and exploratory tendency). Foraging prey had the capacity to both remember and return to good habitat and to remember and avoid predators. In general, we found that forager search time increased and consumption decreased after predator introduction. However, predator habitat domain played a key role in determining how much prey habitat use changed following reintroduction, and the forager's knowledge of alternative habitats and exploratory inclinations affected what types of habitat shifts occurred. Namely, habitat shifts and consumption sacrifices by prey were extreme in some cases, particularly when they were pushed far from their starting locations by broad-domain predators, whereas informed foragers spent less time searching and displayed smaller reductions to consumption than their naïve counterparts following predator exposure. More exploratory foragers exhibited larger habitat shifts, thereby sacrificing consumption but reducing encounters by relocating to refugia, whereas less exploratory foragers managed risk in place and consequently suffered increased encounters while consuming more resources. By implication, reintroductions of predators with broad habitat domains are especially likely to impose foraging and movements costs on prey, but forager spatial memory state can mitigate these effects, as informed foragers can better access alternate habitat and avoid predators with smaller reductions in consumption.

Keywords: habitat domain, behavioral type, predation, memory, individual-based model

\section{INTRODUCTION}

Predators are declining globally in both marine (MacNeil et al., 2020) and terrestrial ecosystems (Ripple et al., 2014). Given the myriad ecosystem services that predators can provide (Estes et al., 2011), these declines have elevated discussion of actions aimed at bolstering predator populations (Ritchie et al., 2012), including efforts to reintroduce endangered or threatened species 
(Fritts et al., 1997; Hayward et al., 2007a), supplementing small relict populations to aid species recovery (Weinberger et al., 2009), managing problematic species and individuals in humananimal conflict scenarios (Linnell et al., 1997; Athreya et al., 2011), and assisted colonization to attempt to prevent extinctions of species threatened by climate change (Shirey and Lamberti, 2010). Notably, however, predator recovery can have undesirable or unanticipated consequences for ecosystems, as when predators prey on or compete with other threatened species (Marshall et al., 2016), negatively impact prey populations (Hayward et al., 2007b; DeCesare et al., 2010), or fail to elicit expected patterns of antipredator behavior (e.g., because of human shielding Muhly et al., 2011). Given the variability of predator restoration outcomes for ecosystems, there is need for studies seeking to illuminate the key factors that mediate competitor and prey responses to recovering predator populations (Alston et al., 2019).

When they perceive predation risk, prey individuals commonly sacrifice food in exchange for the safety afforded by differential space use (e.g., refuging), apprehension, or group size (Lima and Dill, 1990; Preisser et al., 2005; Cresswell, 2008; Say-Sallaz et al., 2019). There is growing recognition, however, that such anti-predator investment can vary in nature and intensity as a function of context, or, in other words, properties of the prey experiencing the danger, the predator imposing the threat, and/or the setting of the interaction (Wirsing et al., 2021). For example, prey energetic state (i.e., body condition or hunger), is known to affect risk-taking behavior by mediating individual differences in the incentive to protect vs. seek assets (energy stores) linked to residual reproductive value (McNamara and Houston, 1986; Lima, 1988; Whitham and Mathis, 2000; Olsson et al., 2002; Heithaus et al., 2007). Accordingly, studies exploring these context-dependent drivers of variation in prey defensive behavior should facilitate more reliable prediction of predator recovery in particular systems.

Memory is a state variable that has often been considered in the context of foraging and migratory decision-making (Bracis and Mueller, 2017; Abrahms et al., 2019; Merkle et al., 2019; Tsalyuk et al., 2019), as supported by experimental evidence (Kamil and Roitblat, 1985; Shettleworth, 2001; Stephens et al., 2007). Animals are known to learn from and subsequently avoid predator encounters (Huntingford and Wright, 1989; Wisenden et al., 1994; Griffin et al., 2000; Nomikou et al., 2003). For example, predator-experienced mice (Mus domesticus) changed their foraging behavior in response to signals of increased predation risk while predator-naïve mice did not, and those differences in foraging behavior correlated with survival (Dickman, 1992). Thus, memory of predator threats as well as memory of alternative foraging locations may both be components of forager state that influence risktaking or aid the forager in managing the food-safety tradeoff. In support of this hypothesis, Bracis et al. (2018) showed that spatial memory of food and risk allowed simulated foragers to reduce predator encounters relative to their naïve counterparts without concomitantly reducing consumption, particularly when predators were persistent and weakly correlated with resources across the modeled landscape. No study to date, however, has explored how memory shapes prey habitat use decisions following the restoration of a predator population.

Memory is particularly challenging to study in an ecological context because it can neither be measured directly nor inferred reliably from detailed information on an animal's past experiences (Fagan et al., 2013; Van Moorter et al., 2013). While spatial familiarity has been shown to be an important driver of resource selection and is suggestive of memory (Wolf et al., 2009), it has also been demonstrated that an apparent but non-existent preference for familiarity can arise when habitat models are incomplete (Van Moorter et al., 2013). Accordingly, modeling, whereby memory state can be controlled explicitly, is better suited to generating insight into how this state variable influences predator-prey interactions. Here, in the interest of moving beyond population dynamics to how broader ecosystem properties may be mediated by interactions between recovering predators and prey (Seddon et al., 2007), we used individual-based modeling to examine the impacts of predator recolonization on prey foraging behavior and, in particular, how memory state with respect to the surrounding landscape shapes subsequent habitat shifts by prey. Thus we specifically examine prey who shift their behavior to avoid predators among many other possible antipredator behaviors (Lima and Dill, 1990). Concretely, we introduced predators to a simulated landscape and varied the size of their habitat domain as a measure of the area they threatened. Habitat domain is specifically the spatial extent over which individuals move while foraging, in contrast to home range which can encompass resources to meet other needs, and can also encompass what available microhabitat is used (Preisser et al., 2007; Schmitz et al., 2017). Foraging prey in these simulations differed in their knowledge of the surrounding landscape and how exploratory they were in the face of new habitat. We analyzed movements of the foragers before and after predators were introduced to the landscape to understand how memory influences changes in the behavioral dimension of space use and how those changes are reflected in consumption and time budget. Under the hypothesis that memory facilitates optimization of the trade-off between food and safety, we predicted that (1) informed foragers would exhibit smaller reductions in consumption and spend less time searching for refuge patches than their naïve counterparts following predator exposure. For all memory states, we also predicted that (2) changes to consumption and space use would increase with predator habitat domain, as greater area threatened should reduce the availability of nearby anti-predator refugia and thus necessitate more search time. Finally, we predicted that (3) exploratory behavior would confer greater plasticity to adapt to newly introduced predators, particularly by enabling larger-scale spatial shifts.

\section{METHODS}

We explored the impact of predator introductions on prey using an existing modeling framework in which foragers move around a dynamic resource landscape, learning patterns of heterogeneity in resources and predator encounters (Bracis 

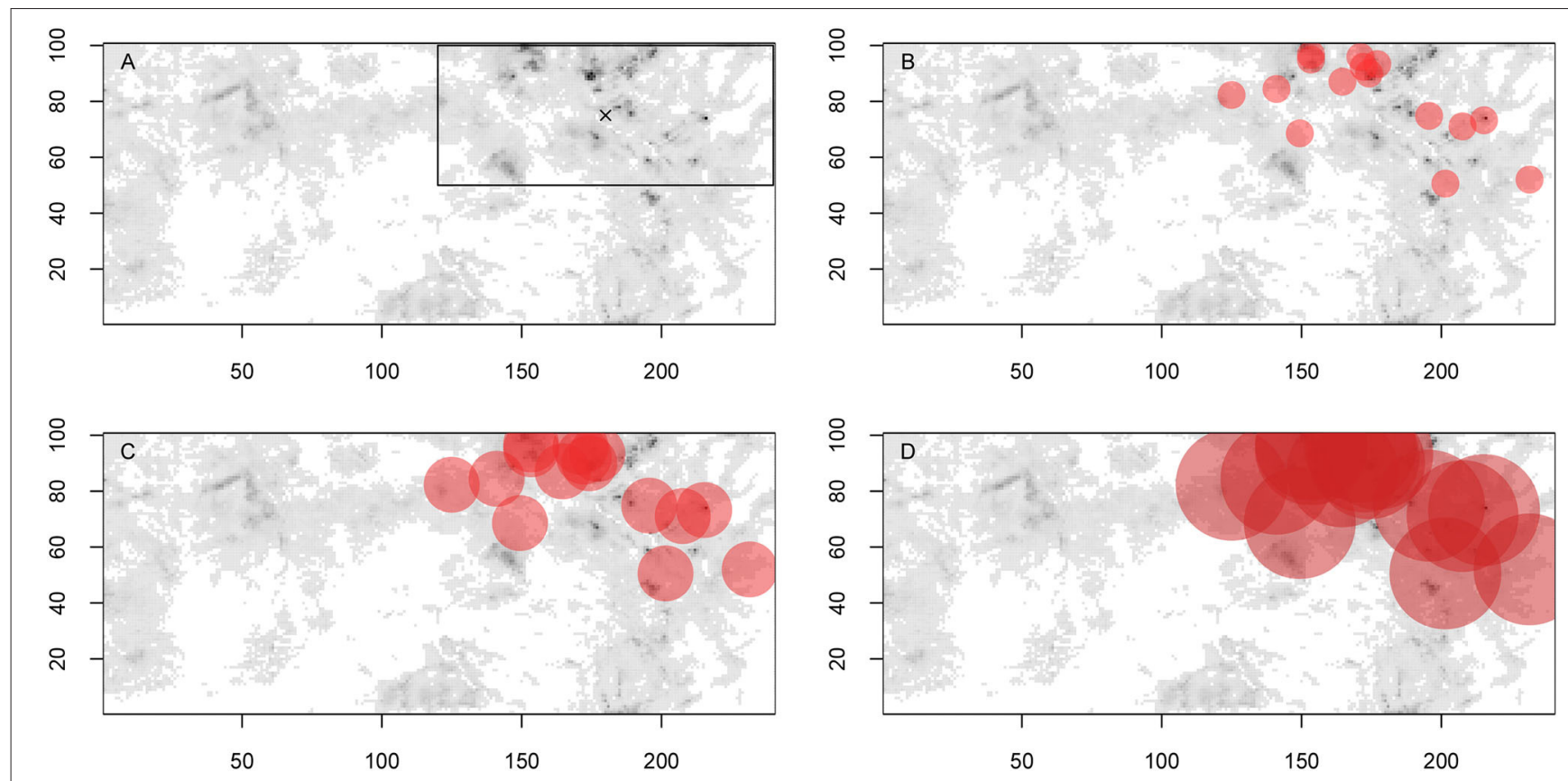

FIGURE 1 | Landscape used in the simulation showing (A) habitat quality and box bounding locations of introduced predators with $\mathbf{x}$ marking the forager's starting location, (B) predators with an encounter radius of 5 , (C) predators with an encounter radius of 10 , and (D) predators with an encounter radius of 20.

et al., 2015, 2018). Foragers continuously consume resources and switch between a more directed, faster searching behavior and a more tortuous, slower feeding behavior as a function of the consumption rate. Note that these predator introductions could represent reintroductions, recolonizations, or colonization, whether naturally occurring or assisted. These foraging resources deplete and regenerate, making it advantageous for the forager to leave recently depleted locations and return to intrinsically high-quality locations. Resource memory takes the form of two different resource streams of information that drive attractive and repulsive tendencies, respectively (Van Moorter et al., 2009; Bracis et al., 2015). One stream drives the forager away from recently visited and depleted areas; the other returns the individual to high-quality areas that have regenerated. We varied how the resource memory is initialized at the beginning of the simulation to compare foragers with complete knowledge of the landscape to those that must learn their surroundings, and we also varied exploratory potential (see below).

Predators appear at particular locations, remain for one quarter of the total simulation time, then disappear according to a Poisson process for timing and location, thus representing sit-and-wait predators, sit-and-pursue predators, or activelyhunting predators with a small home range. Predator locations are correlated with the forager's resource quality (Williams and Flaxman, 2012; Courbin et al., 2014). The forager detects predators within a given encounter radius, then the forager moves directly away from the predator. Predator encounter radius varies to represent predators with different-sized habitat domains. Encounters are tracked, but there is no death, so that all simulations have the same length. The predation memory is a single stream and is a spatially explicit map of predator encounter locations that decays with time (Bracis et al., 2018). Foragers are attracted to good quality habitat while at the same time seeking to minimize predator encounters. The forager selects its direction probabilistically from a circular distribution which is formed by integrating tracts radiating outward from its position of its spatial memory of resource quality discounted by distance, which is then combined with a circular distribution of predator safety.

\subsection{Simulations}

We endeavored to use a landscape with realistic variation in vegetation productivity, but where resources were heterogeneously distributed in space and clumped (Figure 1A). That is, the forager starts in higher productivity habitat before predator introduction and then has the option of remaining in the higher productivity habitat or switching to previously unused lower productivity habitat. Predators are introduced into the highest quality quadrant, matching a common practice of releasing relocated animals in areas of presumed high quality habitat (e.g., Smith and Clark, 1994; Halsey et al., 2015). The distribution of resources is $42 \%$ in the northeast quadrant, $20 \%$ in the southeast quadrant, $14 \%$ in the southwest quadrant, and $24 \%$ in the northwest quadrant. The details of the single landscape used in the simulation can be found in Appendix A.

Simulations begin with no predation, then predators appear in the northeast quadrant of the landscape halfway through the simulation. Within this quadrant, containing the best quality habitat, predator locations are correlated with landscape quality. That is, the probability of a predator being at a location is proportional to that location's quality relative to the quality in the quadrant. Different predator encounter radii (i.e., habitat domains) control how much of the northeast quadrant is 
threatened by predators (Figures 1B-D). This variation in encounter radii affects whether there are refugia within the northeast quadrant once it is occupied by predators. Predators relocate, but rarely (predator duration is half the predator period, see Table B1), to represent relatively stable territories for the introduced predators, while still allowing for some shifts.

Foragers vary in their memory initialization, or memory state. Some foragers start out informed, knowing the spatial pattern of resource quality for the whole landscape. Other foragers start out naïve and have a chance to explore the landscape before predators are introduced. It should be emphasized that "naïve" refers to the forager being unfamiliar with the surrounding habitat, not the predator (Sih et al., 2010). That is, it is assumed that the foragers display effective antipredator behaviors: escape and memory of encounters. Memory state is set at the beginning of the simulation by initializing the two-dimensional spatially explicit slower-decaying attractive resource memory stream, and the faster-decaying repulsive resource memory stream (Bracis et al., 2015). In all cases, the repulsive memory stream that drives foragers away from recently used locations is initialized to zero. For informed foragers, the attractive resource memory stream is initialized to the intrinsic resource quality. For naïve foragers, the attractive resource memory stream is initialized to unvisited expectation parameter $M *$; i.e., how unexplored habitat is valued. We used three different values for how foragers could value unexplored habitat: unexploratory $(M *=0)$, less exploratory $(M *<$ mean quality), and highly exploratory $(M *>$ mean quality), with the average habitat quality roughly halfway between the less and highly exploratory unvisited expectation parameter. In all cases, it is assumed that the foragers know the true average consumption rate of the landscape used to switch between searching and feeding behaviors. Thus, foragers differ in their knowledge of the landscape outside their starting region when predators are introduced and how exploratory they are with new habitat.

Simulations start with the forager located in the center of the northeast quadrant, the area of highest quality, where predators are eventually released (Figure 1A). All parameters used in the simulation are shown in Table B1. Parameters controlling the resource and predation memories that were not varied are set based on the results from Bracis et al. (2018) assuming high survival. We performed 50 replicate simulations for each set of parameters.

\subsection{Metrics}

Foragers' habitat use, consumption, and time budget (i.e., division of time between searching and feeding) were tracked to allow for before-after comparisons of forager behavior with predator introduction. Foragers' consumption is a key metric that provides a measure of the non-consumptive effects of predation (i.e., food given up for safety). The number of predator encounters is also important to contextualize resulting consumption changes after predator introduction and as a measure of risk. Finally, in order to compare the space use before and after predators are introduced, trajectories were visually examined and the utilization distribution was calculated with kernelUD in the adehabitat HR R package (Calenge, 2006).

\section{RESULTS}

\subsection{Space Use Changes}

Space use varied dramatically with memory state, and the kernel density of space use clearly changed after predators were introduced across encounter radii for informed foragers and naïve foragers (Figure 2). How much of the landscape the naive foragers explored before predators were introduced depended on memory state. Namely, unexploratory foragers remained at the high-quality patches closest to the release site, while less exploratory foragers exploited the high-quality areas of the eastern half of the landscape, and highly exploratory foragers began the simulations using the entire landscape. The informed forager's space use most closely resembled that of the less exploratory forager but was more tightly focused on the best patches. After predators were introduced, space use changed across all scenarios as foragers were pushed out of the highestquality areas in the northeast section of the landscape.

Unexploratory foragers (Figure 2, row 2) relocated the least after predator introduction. With the smallest predator encounter radius, these foragers moved out of the immediate vicinity of the predators; the utilization distribution for these foragers closely resembled that for the pre-predator phase as they exploited gaps between predators, with just the upper portion of the distribution shifted eastward. As the encounter radius increased, forager distribution shifted southward but remained in the northeast quadrant. Finally, with the largest encounter radius, forager distribution was completely altered, moving into the southeastern quadrant. Thus, by remaining in the highest-quality quadrant despite predation risk, unexploratory foragers tended to maintain high consumption but at the cost of higher encounters.

Less exploratory foragers showed a stronger shift in habitat use after predator introduction. These foragers (Figure 2, row 3) continued to utilize the better-quality habitat in the gaps between predators when the encounter radius was small, but also expanded into patches to the south and west. This pattern intensified as the predator encounter radius increased, and the forager shifted to predominately using the patches south and west of the predators. The highly exploratory forager (Figure 2, row 4 ), on the other hand, utilized patches across the landscape before predators were introduced, and predators served to move a greater proportion of use to that more distant habitat. With increasing encounter radius, however, all foragers shifted from using the margins around the predators to being nearly completely excluded from most of the northeast quadrant in the vicinity of the predators.

The space use of informed foragers (Figure 2, row 1) was most similar to that of less exploratory foragers in how they relocated in the face of predation. The main difference between informed and naïve foragers was that the informed forager was quicker to exploit more distant patches, even when the encounter radius was small. And while the informed forager also continued to exploit habitat close to predators, it did so to a lesser extent that the naïve foragers, especially as the predator encounter radius became large.

The same patterns can be seen with the full trajectories (Figures B1, B2). Namely, the unexploratory forager was the 


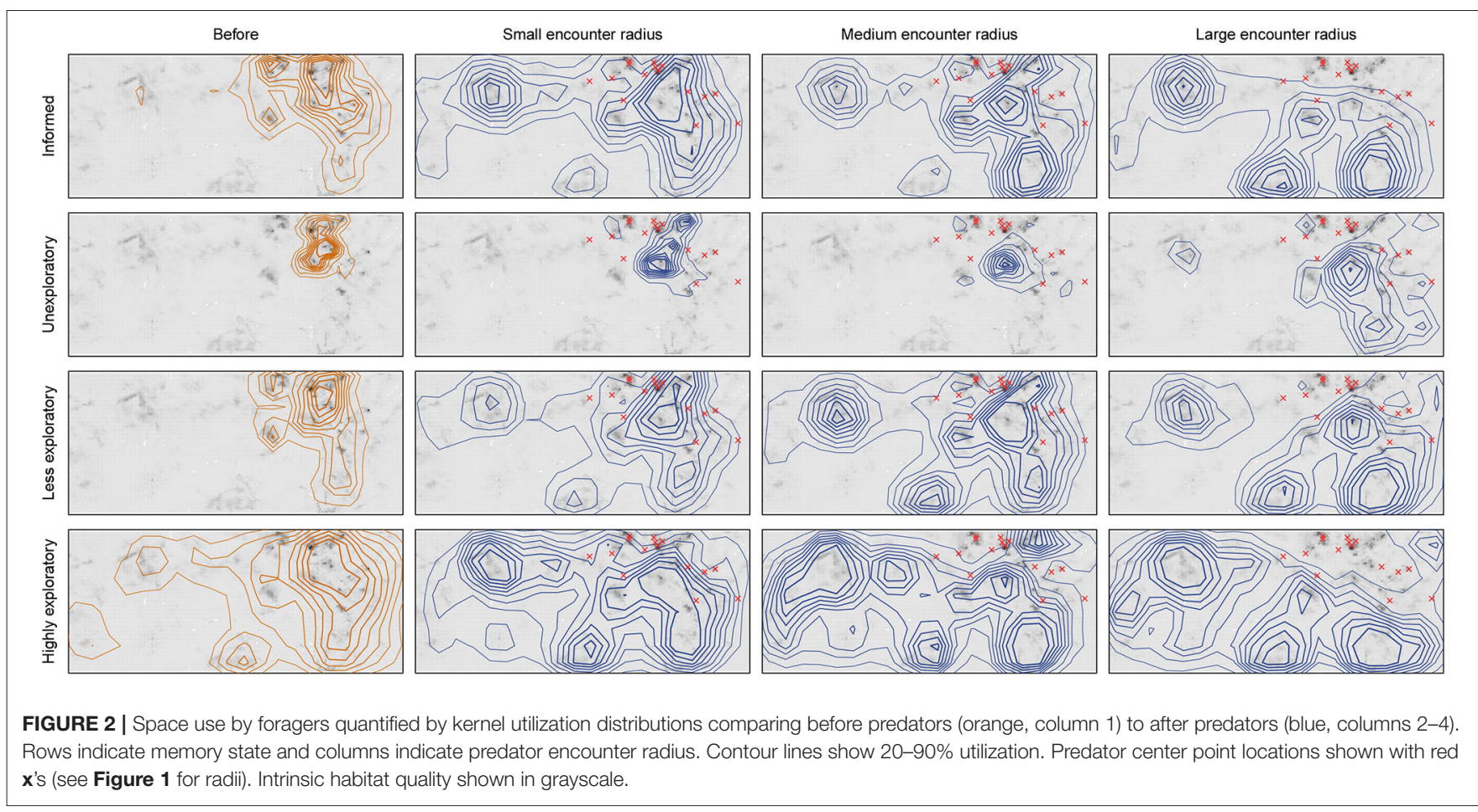

most constrained with large areas of habitat completely unvisited. Whereas the space use patterns of the informed and less exploratory foragers were broadly similar (Figure 2), differences can be seen between the trajectories with the informed forager minimally traveling through unproductive habitat but the less exploratory forager spending more time in unproductive habitat. Finally, the highly exploratory forager had the most dispersed space use across the entire landscape. The partitioning of space by time, with foragers getting pushed out by predators, was clearest with the large predator encounter radius.

\subsection{Consumption Changes}

The integration of space use changes can be seen with the changes in consumption, where predator introductions depressed the amount consumed relative to the period when predators were absent in all scenarios (Figure 3). The forager's memory state partitioned both the pre-predator and post-predator consumption amounts, with higher pre-predator consumption nearly always associated with higher post-predator consumption. Surprisingly, the naïve unexploratory forager consumed the most, followed by the informed forager, the naïve less exploratory forager, and then the naïve highly exploratory forager. Postpredator consumption was mediated by the predator encounter radius, with larger radii associated with larger declines in consumption (Table B3).

In general, higher encounter rates were associated with lower consumption (per memory state), unlike the food-safety tradeoff frequently observed in Bracis et al. (2018). Within a given memory state, larger encounter radii led to higher encounters as more habitat within the most productive quadrant was threatened (Table B3). Naïve unexploratory foragers experienced the highest number of encounters with predators, then informed foragers and naïve less exploratory foragers, followed by naïve highly exploratory foragers.

\subsection{Time Budget and Consumption Rate Changes}

Time spent searching by foragers increased after predators were introduced, driven by foragers leaving their initial habitat to avoid predators and searching for new resources (Figure 4A). This pattern manifested even for informed foragers, which had knowledge of other resource locations but still had to relocate, but was more dramatic for naïve foragers that had to initially locate resources. Overall, increases in search time exhibited the same rank order as those for decreased consumption. The increase in time spent searching was also larger with increasing encounter radius as foragers were more completely displaced from their previous habitat.

In addition to changes in time budget, foragers' consumption rates while feeding declined after predators were introduced (Figure 4B), implying that foragers shifted to lower quality or already depleted habitat. Note that while foragers did consume resources while searching in our simulations, the bulk of consumption ( $\sim 80-90 \%)$ occurred while feeding. Declines in consumption rate were more similar across memory states, with slightly larger declines with larger encounter radii, though this was less true for highly exploratory foragers. Thus, the declines in consumption seen with the introduction of predators were a function of both foragers needing to spend more time searching for food as well as selecting lower quality patches. 

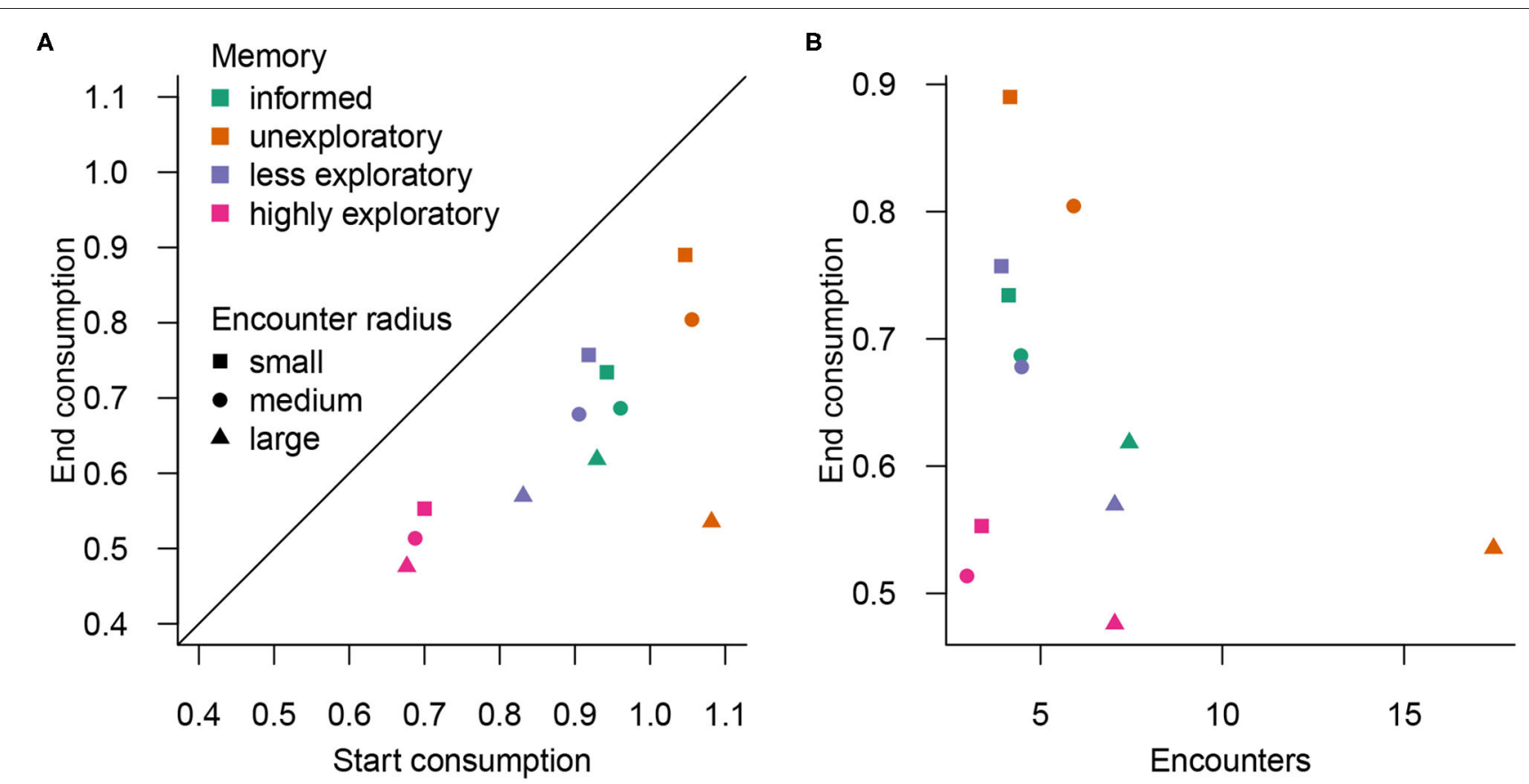

FIGURE 3 | (A) Consumption in the post-predator environment declined in all scenarios compared to the pre-predator environment (i.e., prior to introduction), but was influenced by memory state and encounter radius. (B) Higher encounters generally also led to decreased consumption in the post-predator environment. Memory state is indicated by color and encounter radius by symbol.

\section{DISCUSSION}

Habitat shifts due to changing predation threat have been observed in a wide variety of both terrestrial and aquatic systems (Sih, 1980; Heithaus and Dill, 2002; Wirsing et al., 2007; Belovsky et al., 2011; Dellinger et al., 2019). Yet, previous attempts to predict ecosystem effects of predator reintroductions have neglected prey behavior changes (Baker et al., 2017). Here, to address this information gap, we used individual-based modeling to understand how both predator and prey traits shape behavioral outcomes for foraging prey with the addition of predators to the landscape. Consistent with the non-consumptive effects predators can exert on prey (Preisser et al., 2005), forager behavior, as measured by consumption rates, searching time, and space use, changed after the introduction of predators. The nature of these changes, however, depended on the interplay among the memory state of the forager, the spatial domain threatened by the predator, and the degree to which foragers were exploratory. Namely, in support of our first prediction, informed foragers displayed smaller reductions to consumption and spent less time searching than their naive counterparts following predator exposure. Furthermore, consistent with prediction two, predator habitat domain correlated positively with reductions to consumption and changes to space use, irrespective of memory state. Supporting our third prediction, the degree to which foragers were exploratory shaped their anti-predator responses, with less exploratory prey managing risk in place and consequently suffering increased encounters while consuming more resources, and more exploratory individuals sacrificing consumption but reducing encounters by relocating to refugia. Together, these findings highlight how understanding prey spatial memory and the movement tendencies of both predators and prey is key to predicting the consequences of predator recovery for subsequent prey distribution and fitness.

\subsection{Memory State Influences Prey Response to Predator Recovery}

Spatial memory has been shown to shape habitat selection and movements of translocated animals by facilitating location of high-quality sites (Wolf et al., 2009), and inducing long dispersals from release sites in search of areas that are similar to the translocated individual's natal habitat (Stamps and Swaisgood, 2007). Here, we varied prey memory state in terms of their knowledge of alternative foraging locations in order to explore how it might shape anti-predator responses. Overall, we found prey with all memory states to spend more time searching compared to feeding after predator introduction. Notably, however, changes to consumption differed markedly as a function of memory state. Namely, reflecting the advantage of knowing alternate resource locations, informed foragers exhibited modest increases in search time, and correspondingly minimal reductions in consumption rates, after predator introduction relative to most of their naïve counterparts (but not for naïve unexploratory individuals, see below). This finding suggests that foragers with knowledge of habitat beyond the immediate area threatened by introduced predators might be better adapted to cope with the new threat. By extension, in group-living species that depend on 

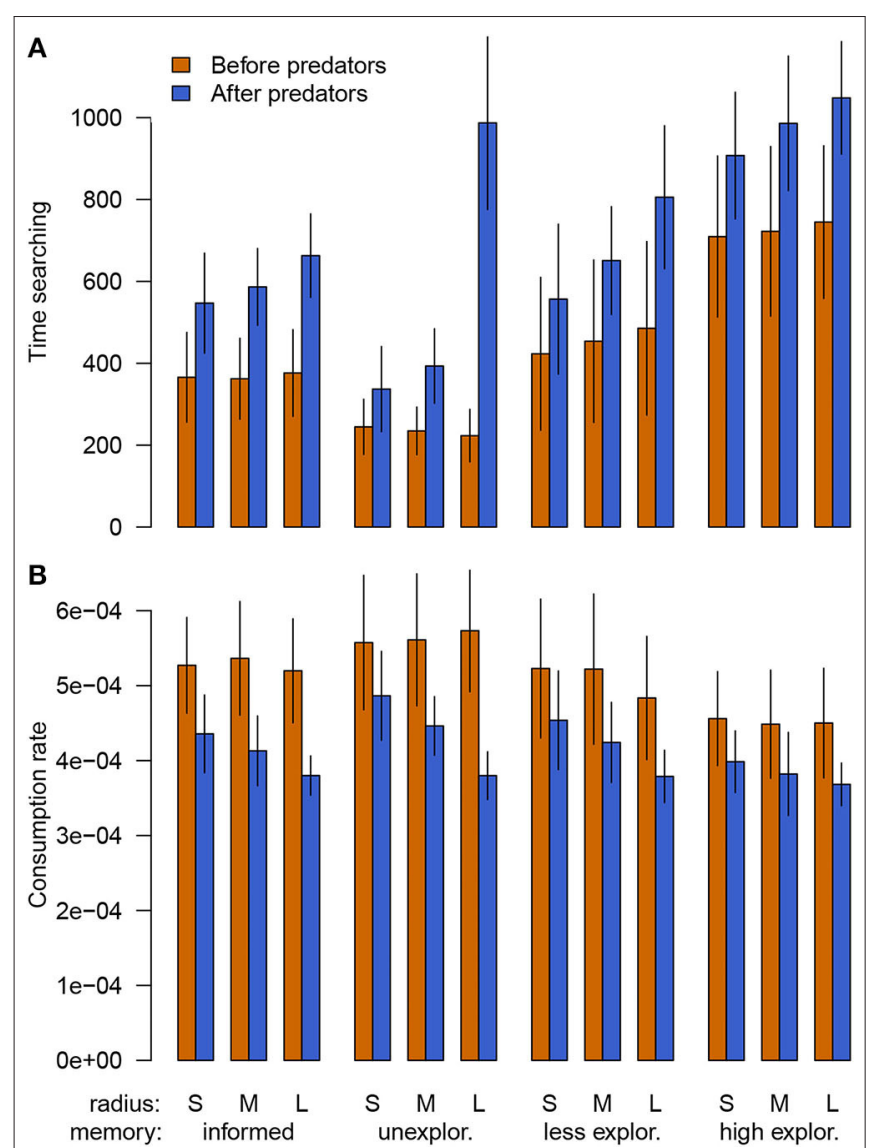

FIGURE 4 | Time budget and consumption rate before and after the introduction of predators across different values for the encounter radius (top line $x$-axis) and for memory state (bottom line $x$-axis) with lines showing the standard deviation. (A) Time spent searching as opposed to feeding (a minimal amount of time was spent in the escape behavior, mean 3-17). (B) Consumption rate while in the feeding behavior.

decision-making by experienced group members to navigate the landscape (e.g., elephants, McComb et al., 2011), the loss of such individuals could affect the vulnerability of the population to predation danger, human and otherwise, disproportionately. Conversely, it may also indicate that introduced predators in this scenario might struggle to find prey. Thus, in accord with the recommendations of Trainor et al. (2014), predicting the success of predator translocation programs may be aided by a functional knowledge of predator-prey interactions that includes the capacity of the potentially affected prey species to relocate to alternate and predator-free foraging areas. Although differentiating between searching and feeding behavior is more challenging in field situations, this is an active area of research (e.g., using movement patterns extracted from GPS locations or accelerometer data; reviewed in Gurarie et al., 2016), thus permitting measurement of changes in time allocation preand post-predator introduction. Accordingly, we may soon be in position to test these hypotheses empirically under field conditions.

\subsection{Predator Domain Determines Degree of Impact on Prey}

Our results suggest that the spatial extent of predator threat, or, in other words, predator habitat domain, influences the impact of predator risk on forager behavior post-introduction. An animal's habitat domain is the part of the available habitat it uses, with broad-domain species that range throughout much of the available space (and time) differing from those with narrow domains that use only some subset of the available space (and/or time) (Preisser et al., 2007). Applying this paradigm to our modeling approach, increasing encounter radius removed potential refugia in the high-quality habitat for prey analogously to predators switching from a narrow-domain to a broaddomain (or to the difference between a narrow- and broaddomain predator in the same system). Not surprisingly, therefore, predators with large domains induced prey to spend more time searching for refugia away from the introduction quadrant and, as a result, to suffer increased penalties to consumption. Interestingly, when also considering forager memory, our findings align broadly with the "hunting mode-habitat domain" concept (Schmitz et al., 2017; Wirsing et al., 2021). Under this framework, prey with domains that extend beyond those of their predators should rely on avoidance to minimize encounters, whereas those whose domains fall within that of a predator are expected to experience more encounters and utilize defenses that reduce the likelihood of death given an encounter. In our modeling scenarios, informed foragers effectively had more immediate access to a habitat domain that extended beyond that of the reintroduced predator, and consequently experienced relatively few predator encounters. By contrast, naïve foragers were less able to escape the domain of the reintroduced predator, being ignorant of more distant refugia, and thus encountered the repatriated predator more frequently. In particular because of their alignment with theory, our modeling results merit evaluation under laboratory and field conditions. Further, the habitat domain concept may also be extended to consider humans as a potential predator, and thus could fit within the framework of how humans and wildlife coexistence, that is the land sparing-land sharing debate (Fischer et al., 2014). For example, an unexploratory forager combined with a small habitat domain predator could lead to land sharing, while either more exploratoriness or a broad habitat domains results in the forager displacing rather than sharing the land, which would require land sparing.

We can also consider habitat domain in the context of the spatial scale of the predator effect. That is, predators with large domains would be expected to exert a stronger selection on where the forager locates it home range, which we saw with larger forager displacements in response to larger domain predators. Habitat selection and how it gives rise to home range has been considered in terms of a hierarchical process of scales, both in space and time. Thus the factor most limiting fitness would be selected at the largest scale, such as large scale avoidance of predation risk and fine scale selection of seasonally available forage (Rettie and Messier, 2000). However, the processes of habitat selection and home range both emerge 
from individual movements, in particular how foragers exploit resources through the amount of time spent and the frequency of visits (Van Moorter et al., 2016). As a consequence of this, habitat selection at a given scale is driven not only by the most limiting factor at that scale, but also by which is the most variable (Van Moorter et al., 2016). Indeed, our results show that the scale of predator avoidance (i.e., scale of habitat selection: 2nd vs. 3rd order according to Johnson, 1980) is driven by the scale of variation in predation risk (i.e., predator habitat domain): a large habitat domain leads to home range displacement, whereas a small habitat domain results in smaller shifts in foraging patches.

\subsection{Exploratory Tendency Controls Size of Habitat Shift}

Individuals can differ consistently along several behavioral axes including activity, aggressiveness, exploration-avoidance, shyness-boldness, and sociability (Réale et al., 2007). These persistent behavioral differences, or behavioral types, within populations can have important ecological implications (Sih et al., 2012), For example, in free-ranging elk (Cervus canadensis) introduced to a novel environment, dispersal distance and time varied by individual, with social individuals being more sedentary than solitary conspecifics (Fryxell et al., 2008). Our modeling results reveal that individual differences in exploratory tendency can shape prey movements following predator reintroduction or colonization in ways that influence encounter rates and, by extension, the probability of predator-inflicted mortality (Lima and Dill, 1990). Namely, highly exploratory foragers were minimally affected by degree of predator threat, as they tended to have the most wide-ranging movements and were less likely to remain in the initial release location when the predators were introduced. By contrast, unexploratory foragers tended to remain in the same area despite the introduction of predation risk, leading to higher consumption but also high encounters, a food-safety trade-off also observed in other contexts (Bracis et al., 2018). When predators were confined to a small portion of the landscape, this tendency allowed naïve unexploratory foragers to achieve higher consumption than even informed foragers. However, when predator search radii expanded such that informed and exploratory foragers were pushed out of the high quality and forced to seek new areas, naïve unexploratory foragers performed poorly from a fitness perspective, exhibiting both depressed consumption rates (necessitated by locally searching for refugia) and extremely high encounters. This pattern may help explain why spatial responses are not universally observed after predator reintroductions (Davies et al., 2016). It also highlights the role exploratory tendency appears to play in mediating how naïve prey individuals respond to, and are affected by, restored predator populations as a focus for empirical investigation. Notably, being exploratory may also help prey to regularly refresh their spatial understanding of recent resource changes and thus to optimize space use decisions in the face of predation risk. Here we held resource quality constant, leaving future work to explore the interplay among predator introduction, prey memory and exploratory tendency, and resource dynamics.

\subsection{Future Perspectives}

Most work on animal movement continues to focus on external factors rather than underlying processes (Joo et al., 2020). By contrast, as memory is likely key to understanding patterns observed in animal foraging (Fagan et al., 2013) and thus an emerging area of research (e.g., Avgar et al., 2013; Bracis et al., 2015; Merkle et al., 2017), we utilize a cognitive paradigm to provide an mechanistic understanding of how animals make movement decisions. Our current work utilizes a flexible modeling framework for exploring how memory can modulate a forager's response to predator introductions, including individual differences in exploratory tendency and habitat knowledge. However, our model utilizes a simple food web comprising the resource, the prey, and the predator. Extending the model to include conspecific interactions or additional predators could provide additional insight, particularly for predator introductions in ecosystems with more complex community structure. For example, multiple predators sharing a habitat domain can reduce the predation risk experienced by the prey, an important consideration for multi-predator systems (Woodcock and Heard, 2011). Other conspecifics may limit where a forager could relocate, particularly for territorial animals (Stamps, 1991; Potts et al., 2012). Here, we examine habitat changes in response to predation, but other antipredator behaviors are possible (e.g., increased vigilance, counter-attack, herd behavior, etc.). Another possible model extension would be to consider different predator hunting modes, such as active hunting vs. ambush/stalking, to examine how this variability affects forager responses. This could include allowing predators to relocate dynamically in response to prey behavior rather than being located solely in response to the prey's resource quality as in the current model. Notably, we differentiated between naïve and informed foragers, but individuals in both of these categories could also differ with respect to the scales at which they mentally map both resources and predation risk. Accordingly, a fruitful avenue for future simulation work in this area would be to explore how foragers' behavior for a given memory state is shaped by the scale of their mental map, before and after predator introduction. Finally, considering dynamic resources (where their intrinsic quality changes) would make it possible to situate this work within the context of environmental change, whether due to habitat loss or climate change.

\section{CONCLUSION}

In conclusion, our modeling exercise reveals that predator introductions can change habitat usage and consumption rates of foragers to varying degrees that depend on the area threatened by the predator and thus how critical it is to avoid encounters. When foragers do shift habitat use in response to predator introductions, memory state (habitat knowledge) and exploratory inclination (behavioral type) mediate how foragers use alternative habitats and experience changes to consumption rates and predator encounters. Search time increased and consumption decreased after predator introduction across all memory states. For foragers with full knowledge of the landscape, 
the increase in search time was relatively modest, reflecting the advantage of knowing alternate resource locations. Thus, spatial memory of the surrounding area can mitigate the effect of introduced predators, as foragers can better access alternate habitat refugia. Notably, forager naïveté was costly in terms of the time needed to find refugia, and prey individuals that were both naïve and unexploratory suffered reduced consumption rates and high predator encounter rates. Potential changes in foraging behavior are an important, though often neglected, component of predator reintroductions, given the far-reaching ecological consequences of top-predator losses (Estes et al., 2011). While memory and individual behavioral variation are challenging to consider in experimental studies of reintroduction (Fagan et al., 2013), evidence from modeling here suggests that dynamic interplay among these two factors and key predator traits (habitat domain) is a critical driver of how forager behavior changes.

\section{DATA AVAILABILITY STATEMENT}

The raw data supporting the conclusions of this article will be made available by the authors, without undue reservation.

\section{REFERENCES}

Abrahms, B., Hazen, E. L., Aikens, E. O., Savoca, M. S., Goldbogen, J. A., Bograd, S. J., et al. (2019). Memory and resource tracking drive blue whale migrations. Proc. Natl. Acad. Sci. U.S.A. 116, 5582-5587. doi: 10.1073/pnas.1819031116

Alston, J., Maitland, B., Brito, B., Esmaeili, S., Ford, A., Hays, B., et al. (2019). Reciprocity in restoration ecology: When might large carnivore reintroduction restore ecosystems? Biol. Conserv. 234, 82-89. doi: 10.1016/j.biocon.2019.03.021

Athreya, V., Odden, M., Linnell, J. D. C., and Karanth, K. U. (2011). Translocation as a tool for mitigating conflict with leopards in human-dominated landscapes of India. Conserv. Biol. 25, 133-141. doi: 10.1111/j.1523-1739.2010.01599.x

Avgar, T., Deardon, R., and Fryxell, J. M. (2013). An empirically parameterized individual based model of animal movement, perception, and memory. Ecol. Model. 251, 158-172. doi: 10.1016/j.ecolmodel.2012.12.002

Baker, C. M., Gordon, A., and Bode, M. (2017). Ensemble ecosystem modeling for predicting ecosystem response to predator reintroduction. Conserv. Biol. 31, 376-384. doi: 10.1111/cobi.12798

Belovsky, G. E., Laws, A. N., and Slade, J. B. (2011). Prey change behaviour with predation threat, but demographic effects vary with prey density: experiments with grasshoppers and birds. Ecol. Lett. 14, 335-340. doi: 10.1111/j.1461-0248.2011.01591.x

Bracis, C., Gurarie, E., Rutter, J., and Goodwin, R. A. (2018). Remembering the good and the bad: memory-based mediation of the food-safety trade-off in dynamic landscapes. Theor. Ecol. 11, 305-319. doi: 10.1007/s12080-018-0367-2

Bracis, C., Gurarie, E., Van Moorter, B., and Goodwin, R. A. (2015). Memory effects on movement behavior in animal foraging. PLoS ONE 10:e136057. doi: 10.1371/journal.pone.0136057

Bracis, C., and Mueller, T. (2017). Memory, not just perception, plays an important role in terrestrial mammalian migration. Proc. R. Soc. B Biol. Sci. 284:20170449. doi: $10.1098 / \mathrm{rspb} .2017 .0449$

Calenge, C. (2006). The package adehabitat for the $\mathrm{R}$ software: tool for the analysis of space and habitat use by animals. Ecol. Model. 197:1035. doi: 10.1016/j.ecolmodel.2006.03.017

Courbin, N., Fortin, D., Dussault, C., and Courtois, R. (2014). Logging-induced changes in habitat network connectivity shape behavioral interactions in the wolf-caribou-moose system. Ecol. Monogr. 84, 265-285. doi: 10.1890/12-2118.1

Cresswell, W. (2008). Non-lethal effects of predation in birds. Ibis 150, 3-17. doi: 10.1111/j.1474-919X.2007.00793.x

Davies, A. B., Tambling, C. J., Kerley, G. I., and Asner, G. P. (2016). Limited spatial response to direct predation risk by African herbivores

\section{AUTHOR CONTRIBUTIONS}

$\mathrm{CB}$ designed and executed the study. $\mathrm{CB}$ and $\mathrm{AW}$ interpreted the results and wrote the paper.

\section{FUNDING}

CB was partially funded through an ERDC BAA at the University of Washington.

\section{ACKNOWLEDGMENTS}

The authors wold like to thank Eliezer Gurarie and R. Andrew Goodwin for helpful comments on an earlier version of this manuscript as well as two reviewers for their comments.

\section{SUPPLEMENTARY MATERIAL}

The Supplementary Material for this article can be found online at: https://www.frontiersin.org/articles/10.3389/fevo. 2021.698370/full\#supplementary-material

following predator reintroduction. Ecol. Evol. 6, 5728-5748. doi: 10.1002/ece3. 2312

DeCesare, N. J., Hebblewhite, M., Robinson, H. S., and Musiani, M. (2010). Endangered, apparently: the role of apparent competition in endangered species conservation. Anim. Conserv. 13, 353-362. doi: 10.1111/j.1469-1795.2009.00328.x

Dellinger, J. A., Shores, C. R., Craig, A., Heithaus, M. R., Ripple, W. J., and Wirsing, A. J. (2019). Habitat use of sympatric prey suggests divergent antipredator responses to recolonizing gray wolves. Oecologia 189, 487-500. doi: 10.1007/s00442-018-4323-z

Dickman, C. R. (1992). Predation and habitat shift in the house mouse, Mus domesticus. Ecology 73, 313-322. doi: 10.2307/1938742

Estes, J. A., Terborgh, J., Brashares, J. S., Power, M. E., Berger, J., Bond, W. J., et al. (2011). Trophic downgrading of planet earth. Science 333, 301-306. doi: $10.1126 /$ science.1205106

Fagan, W. F., Lewis, M. A., Auger-Méthé, M., Avgar, T., Benhamou, S., Breed, G., et al. (2013). Spatial memory and animal movement. Ecol. Lett. 16, 1316-1329. doi: $10.1111 /$ ele. 12165

Fischer, J., Abson, D. J., Butsic, V., Chappell, M. J., Ekroos, J., Hanspach, J., et al. (2014). Land sparing versus land sharing: moving forward. Conserv. Lett. 7, 149-157. doi: 10.1111/conl.12084

Fritts, S. H., Bangs, E. E., Fontaine, J. A., Johnson, M. R., Phillips, M. K., Koch, E. D., et al. (1997). Planning and implementing a reintroduction of wolves to Yellowstone National Park and central Idaho. Restor. Ecol. 5, 7-27. doi: 10.1046/j.1526-100X.1997.09702.x

Fryxell, J. M., Hazell, M., Börger, L., Dalziel, B. D., Haydon, D. T., Morales, J. M., et al. (2008). Multiple movement modes by large herbivores at multiple spatiotemporal scales. Proc. Natl. Acad. Sci. U.S.A 105, 19114-19119. doi: 10.1073/pnas.0801737105

Griffin, A. S., Blumstein, D. T., and Evans, C. S. (2000). Training captive-bred or translocated animals to avoid predators. Conserv. Biol. 14, 1317-1326. doi: 10.1046/j.1523-1739.2000.99326.x

Gurarie, E., Bracis, C., Delgado, M., Meckley, T. D., Kojola, I., and Wagner, C. M. (2016). What is the animal doing? Tools for exploring behavioural structure in animal movements. J. Anim. Ecol. 85, 69-84. doi: 10.1111/1365-2656. 12379

Halsey, S. M., Zielinski, W. J., and Scheller, R. M. (2015). Modeling predator habitat to enhance reintroduction planning. Landsc. Ecol. 30, 1257-1271. doi: 10.1007/s10980-015-0177-5

Hayward, M. W., Kerley, G. I. H., Adendorff, J., Moolman, L. C., O’Brien, J., Sholto-Douglas, A., et al. (2007a). The reintroduction of large carnivores 
to the Eastern Cape, South Africa: an assessment. Oryx 41, 205-214. doi: 10.1017/S0030605307001767

Hayward, M. W., O'Brien, J., and Kerley, G. I. (2007b). Carrying capacity of large African predators: predictions and tests. Biol. Conserv. 139, 219-229. doi: 10.1016/j.biocon.2007.06.018

Heithaus, M. R., and Dill, L. M. (2002). Food availability and tiger shark predation risk influence bottlenose dolphin habitat use. Ecology 83, 480-491. doi: 10.1890/0012-9658(2002)083[0480:FAATSP]2.0.CO;2

Heithaus, M. R., Frid, A., Wirsing, A. J., Dill, L. M., Fourqurean, J. W., Burkholder, D., et al. (2007). State-dependent risk-taking by green sea turtles mediates topdown effects of tiger shark intimidation in a marine ecosystem. J. Anim. Ecol. 76, 837-844. doi: 10.1111/j.1365-2656.2007.01260.x

Huntingford, F., and Wright, P. (1989). How sticklebacks learn to avoid dangerous feeding patches. Behav. Process. 19, 181-189. doi: 10.1016/0376-6357(89)90040-5

Johnson, D. H. (1980). The comparison of usage and availability measurements for evaluating resource preference. Ecology 61, 65-71. doi: 10.2307/1937156

Joo, R., Picardi, S., Boone, M. E., Clay, T. A., Patrick, S. C., Romero-Romero, V. S., et al. (2020). A decade of movement ecology. arXiv [preprint] arXiv:2006.00110. https://arxiv.org/abs/2006.00110

Kamil, A. C., and Roitblat, H. L. (1985). The ecology of foraging behavior: implications for animal learning and memory. Annu. Rev. Psychol. 36, 141-169. doi: 10.1146/annurev.ps.36.020185.001041

Lima, S. L. (1988). Initiation and termination of daily feeding in dark-eyed juncos: influences of predation risk and energy reserves. Oikos 53, 3-11. doi: $10.2307 / 3565656$

Lima, S. L., and Dill, L. M. (1990). Behavioral decisions made under the risk of predation: a review and prospectus. Can. J. Zool. 68, 619-640. doi: 10.1139/z90-092

Linnell, J. D., Aanes, R., Swenson, J. E., Odden, J., and Smith, M. E. (1997). Translocation of carnivores as a method for managing problem animals: a review. Biodivers. Conserv. 6, 1245-1257. doi: 10.1023/B:BIOC.0000034011.05412.cd

MacNeil, M. A., Chapman, D. D., Heupel, M., Simpfendorfer, C. A., Heithaus, M., Meekan, M., et al. (2020). Global status and conservation potential of reef sharks. Nature 583, 801-806. doi: 10.1038/s41586-020-2519-y

Marshall, K. N., Stier, A. C., Samhouri, J. F., Kelly, R. P., and Ward, E. J. (2016). Conservation challenges of predator recovery. Conserv. Lett. 9, 70-78. doi: $10.1111 /$ conl.12186

McComb, K., Shannon, G., Durant, S. M., Sayialel, K., Slotow, R., Poole, J., et al. (2011). Leadership in elephants: the adaptive value of age. Proc. R. Soc. B Biol. Sci. 278, 3270-3276. doi: 10.1098/rspb.2011.0168

McNamara, J. M., and Houston, A. I. (1986). The common currency for behavioral decisions. Am. Nat. 127, 358-378. doi: 10.1086/284489

Merkle, J. A., Potts, J. R., and Fortin, D. (2017). Energy benefits and emergent space use patterns of an empirically parameterized model of memory- based patch selection. Oikos 126. doi: 10.1111/oik.03356

Merkle, J. A., Sawyer, H., Monteith, K. L., Dwinnell, S. P. H., Fralick, G. L., and Kauffman, M. J. (2019). Spatial memory shapes migration and its benefits: evidence from a large herbivore. Ecol. Lett. 22, 1797-1805. doi: $10.1111 /$ ele.13362

Muhly, T. B., Semeniuk, C., Massolo, A., Hickman, L., and Musiani, M. (2011). Human activity helps prey win the predator-prey space race. PLoS ONE 6:e17050. doi: 10.1371/journal.pone.0017050

National Research Council (2002). Ecological Dynamics on Yellowstone's Northern Range. National Academies Press.

Nomikou, M., Janssen, A., and Sabelis, M. (2003). Herbivore host plant selection: whitefly learns to avoid host plants that harbour predators of her offspring. Oecologia 136, 484-488. doi: 10.1007/s00442-003-1289-1

Olsson, O., Brown, J. S., and Smith, H. G. (2002). Long- and short-term statedependent foraging under predation risk: an indication of habitat quality. Anim. Behav. 63, 981-989. doi: 10.1006/anbe.2001.1985

Potts, J. R., Harris, S., and Giuggioli, L. (2012). Territorial dynamics and stable home range formation for central place foragers. PLoS ONE 7:e34033. doi: 10.1371/journal.pone.0034033

Preisser, E. L., Bolnick, D. I., and Benard, M. F. (2005). Scared to death? The effects of intimidation and consumption in predator-prey interactions. Ecology 86, 501-509. doi: 10.1890/04-0719
Preisser, E. L., Orrock, J. L., and Schmitz, O. J. (2007). Predator hunting mode and habitat domain alter nonconsumptive effects in predator-prey interactions. Ecology 88, 2744-2751. doi: 10.1890/07-0260.1

Réale, D., Reader, S. M., Sol, D., McDougall, P. T., and Dingemanse, N. J. (2007). Integrating animal temperament within ecology and evolution. Biol. Rev. 82, 291-318. doi: 10.1111/j.1469-185X.2007.00010.x

Rettie, W. J., and Messier, F. (2000). Hierarchical habitat selection by woodland caribou: its relationship to limiting factors. Ecography 23, 466-478. doi: 10.1111/j.1600-0587.2000.tb00303.x

Ripple, W. J., Estes, J. A., Beschta, R. L., Wilmers, C. C., Ritchie, E. G., Hebblewhite, M., et al. (2014). Status and ecological effects of the world's largest carnivores. Science 343:1241484. doi: 10.1126/science.1241484

Ritchie, E. G., Elmhagen, B., Glen, A. S., Letnic, M., Ludwig, G., and McDonald, R. A. (2012). Ecosystem restoration with teeth: what role for predators? Trends Ecol. Evol. 27, 265-271. doi: 10.1016/j.tree.2012.01.001

Say-Sallaz, E., Chamaillé-Jammes, S., Fritz, H., and Valeix, M. (2019). Nonconsumptive effects of predation in large terrestrial mammals: mapping our knowledge and revealing the tip of the iceberg. Biol. Conserv. 235, 36-52. doi: 10.1016/j.biocon.2019.03.044

Schmitz, O. J., Miller, J. R. B., Trainor, A. M., and Abrahms, B. (2017). Toward a community ecology of landscapes: predicting multiple predatorprey interactions across geographic space. Ecology 98, 2281-2292. doi: 10.1002/ecy.1916

Seddon, P. J., Armstrong, D. P., and Maloney, R. F. (2007). Developing the science of reintroduction biology. Conserv. Biol. 21, 303-312. doi: $10.1111 /$ j.1523-1739.2006.00627.x

Shettleworth, S. J. (2001). Animal cognition and animal behaviour. Anim. Behav. 61, 277-286. doi: 10.1006/anbe.2000.1606

Shirey, P. D, and Lamberti, G. A. (2010). Assisted colonization under the U.S. Endangered Species Act. Conserv. Lett. 3, 45-52. doi: 10.1111/j.1755-263X.2009.00083.x

Sih, A. (1980). Optimal behavior: can foragers balance two conflicting demands? Science 210, 1041-1043. doi: 10.1126/science.210.4473.1041

Sih, A., Bolnick, D. I., Luttbeg, B., Orrock, J. L., Peacor, S. D., Pintor, L. M., et al. (2010). Predator-prey naïveté, antipredator behavior, and the ecology of predator invasions. Oikos 119, 610-621. doi: 10.1111/j.1600-0706.2009.18039.x

Sih, A., Cote, J., Evans, M., Fogarty, S., and Pruitt, J. (2012). Ecological implications of behavioural syndromes. Ecol. Lett. 15, 278-289. doi: 10.1111/j.1461-0248.2011.01731.x

Smith, K. G., and Clark, J. D. (1994). Black bears in Arkansas: characteristics of a successful translocation. J. Mammal. 75, 309-320. doi: 10.2307/1382549

Stamps, J. (1991). The effect of conspecifics on habitat selection in territorial species. Behav. Ecol. Sociobiol. 28, 29-36. doi: 10.1007/BF00172136

Stamps, J. A., and Swaisgood, R. R. (2007). Someplace like home: experience, habitat selection and conservation biology. Appl. Anim. Behav. Sci. 102, 392-409. doi: 10.1016/j.applanim.2006.05.038

Stephens, D. W., Brown, J. S., and Ydenberg, R. C., editors (2007). Foraging: Behavior and Ecology. Chicago, IL: University of Chicago Press. doi: 10.7208/chicago/9780226772653.001.0001

Trainor, A. M., Schmitz, O. J., Ivan, J. S., and Shenk, T. M. (2014). Enhancing species distribution modeling by characterizing predator-prey interactions. Ecol. Appl. 24, 204-216. doi: 10.1890/13-0336.1

Tsalyuk, M., Kilian, W., Reineking, B., and Getz, W. M. (2019). Temporal variation in resource selection of African elephants follows long-term variability in resource availability. Ecol. Monogr. 89:e01348. doi: 10.1002/ecm.1348

Van Moorter, B., Rolandsen, C. M., Basille, M., and Gaillard, J. (2016). Movement is the glue connecting home ranges and habitat selection. J. Anim. Ecol. 85, 21-31. doi: 10.1111/1365-2656.12394

Van Moorter, B., Visscher, D., Benhamou, S., Börger, L., Boyce, M. S., and Gaillard, J.-M. (2009). Memory keeps you at home: a mechanistic model for home range emergence. Oikos 118, 641-652. doi: 10.1111/j.1600-0706.2008. 17003.x

Van Moorter, B., Visscher, D., Herfindal, I., Basille, M., and Mysterud, A. (2013). Inferring behavioural mechanisms in habitat selection studies getting the null-hypothesis right for functional and familiarity responses. Ecography 36, 323-330. doi: 10.1111/j.1600-0587.2012.07291.x

Weinberger, I. C., Bontadina, F., and Arlettaz, R. (2009). Translocation as a conservation tool to supplement relict bat colonies: a pioneer 
study with endangered horseshoe bats. Endanger. Species Res. 8, 41-48. doi: 10.3354/esr00196

Whitham, J., and Mathis, A. (2000). Effects of hunger and predation risk on foraging behavior of graybelly salamanders, Euryceaurycea multiplicata. J. Chem. Ecol. 26, 1659-1665. doi: 10.1023/A:10055909 13680

Williams, A. C., and Flaxman, S. M. (2012). Can predators assess the quality of their prey's resource? Anim. Behav. 83, 883-890. doi: 10.1016/j.anbehav.2012. 01.008

Wirsing, A. J., Heithaus, M. R., Brown, J. S., Kotler, B. P., and Schmitz, O. J. (2021). The context dependence of non-consumptive predator effects. Ecol. Lett. 24, 113-129. doi: 10.1111/ele.13614

Wirsing, A. J., Heithaus, M. R., and Dill, L. M. (2007). Fear factor: do dugongs (Dugong dugon) trade food for safety from tiger sharks (Galeocerdo cuvier)? Oecologia 153, 1031-1040. doi: 10.1007/s00442-007-0802-3

Wisenden, B. D., Chivers, D. P., and Smith, R. J. F. (1994). Risk-sensitive habitat use by brook stickleback (Culaea inconstans) in areas associated with minnow alarm pheromone. J. Chem. Ecol. 20, 2975-2983. doi: 10.1007/BF020 98403

Wolf, M., Frair, J., Merrill, E., and Turchin, P. (2009). The attraction of the known: the importance of spatial familiarity in habitat selection in wapiti Cervus elaphus. Ecography 32, 401-410. doi: 10.1111/j.1600-0587.2008.05626.x
Woodcock, B. A., and Heard, M. S. (2011). Disentangling the effects of predator hunting mode and habitat domain on the top-down control of insect herbivores. J. Anim. Ecol. 80, 495-503. doi: 10.1111/j.1365-2656.2010. 01790.x

Conflict of Interest: The authors declare that the research was conducted in the absence of any commercial or financial relationships that could be construed as a potential conflict of interest.

Publisher's Note: All claims expressed in this article are solely those of the authors and do not necessarily represent those of their affiliated organizations, or those of the publisher, the editors and the reviewers. Any product that may be evaluated in this article, or claim that may be made by its manufacturer, is not guaranteed or endorsed by the publisher.

Copyright (c) 2021 Bracis and Wirsing. This is an open-access article distributed under the terms of the Creative Commons Attribution License (CC BY). The use, distribution or reproduction in other forums is permitted, provided the original author(s) and the copyright owner(s) are credited and that the original publication in this journal is cited, in accordance with accepted academic practice. No use, distribution or reproduction is permitted which does not comply with these terms. 